\title{
Le battage dans la fabrication des céramiques coréennes à l'Âge du Fer
}

Laurence Denès

\section{(2) OpenEdition}

12 Journals

Édition électronique

URL : https://journals.openedition.org/tc/99

DOI : $10.4000 /$ tc. 99

ISSN : 1952-420X

Éditeur

Éditions de l'EHESS

\section{Édition imprimée}

Date de publication : 1 avril 2004

Pagination : 43-75

ISSN : 0248-6016

\section{Référence électronique}

Laurence Denès, «Le battage dans la fabrication des céramiques coréennes à l'Âge du Fer »,

Techniques \& Culture [En ligne], 42 | 2004, mis en ligne le 06 novembre 2007, consulté le 29 septembre 2022. URL : http://journals.openedition.org/tc/99 ; DOI : https://doi.org/10.4000/tc.99

Ce document a été généré automatiquement le 29 septembre 2022.

Tous droits réservés 


\title{
Le battage dans la fabrication des céramiques coréennes à l'Âge du Fer
}

\author{
Laurence Denès
}

1 La technique du battage permet, à partir d'une ébauche, de mettre en forme la panse et la base des vases, voire aussi le col et la lèvre, en frappant la paroi entre un battoir et un contre-battoir. Lorsque le battoir est gravé ou entouré de cordes, cela produit une sorte de décor imprimé couvrant toute la surface travaillée. Le martèlement de la surface peut également servir, de façon plus ponctuelle, à retoucher une forme, à fermer la base des récipients ou encore à souder deux parties fabriquées séparément (Gibson \& Woods 1990 : 95-96; van der Leeuw 1984 : 333).

2 Dans le sud-ouest de la Corée, le battage a été utilisé pour le façonnage et le décor des céramiques -on parle ainsi de " céramiques à décor au battoir »- à partir du début de notre ère, environ quatre siècles après son introduction dans le nord de la péninsule. Intimement liée ici à la culture de l'Âge du Fer, elle a été abandonnée vers les Ve-VIe siècles, parallèlement à l'extension du royaume de Paekche vers le sud (fig. 1). 
Figure 1. Carte générale du sud de la péninsule coréenne. Situation aux Ille-IVe siècles. Paekche, Silla et Kaya représentent les trois premiers royaumes du sud de la péninsule (environ Ille-VIle siècles)

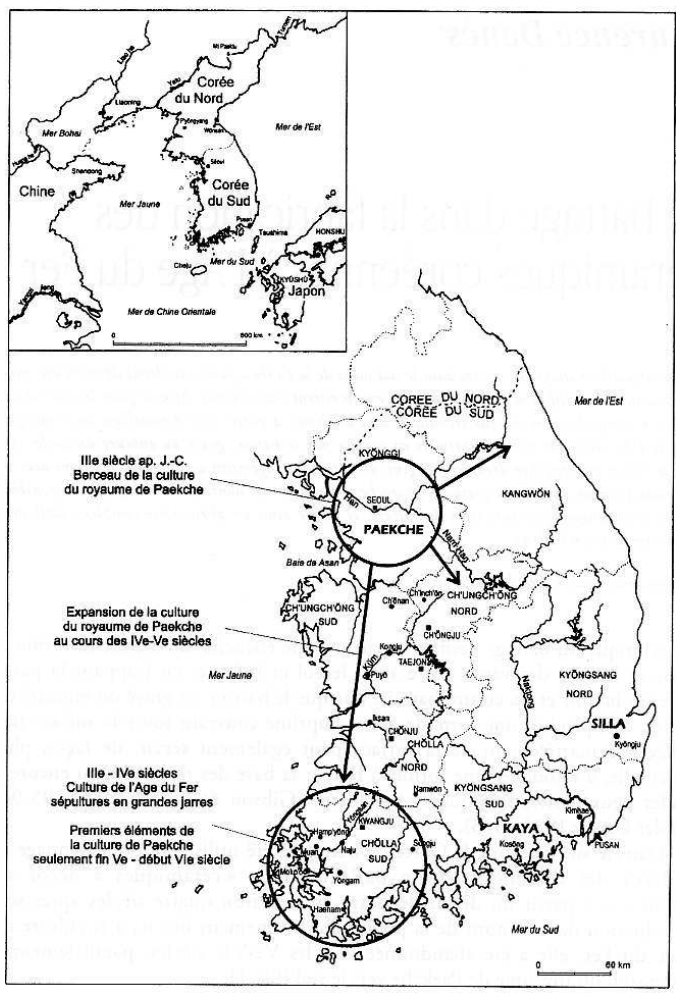

3 Au cours des premiers siècles de notre ère, la production céramique du sud-ouest de la péninsule est également marquée par l'apparition de nouveaux types de fours et par un nombre important de pièces défectueuses (déformations, boursouflures...).

Dans le cadre de cet article $^{1}$, qui ne constitue qu'une première approche de la technique du battage en Corée, je montrerai tout d'abord que plusieurs procédés de façonnage de la base des récipients peuvent être distingués au sein de la catégorie des céramiques dites « à décor au battoir ». À partir de ces données seront appréhendées les grandes lignes de l'évolution du battage dans le sud-ouest de la péninsule. J'évoquerai également les changements occasionnés par l'adoption de cette nouvelle technique et nous verrons quelles peuvent être les raisons de son utilisation massive. Enfin, le problème du degré de maîtrise technique des potiers du sud-ouest de la Corée pendant l'Âge du Fer sera abordé, comme les relations entre le battage et les nouveaux types de four : même origine ? évolution parallèle?

Cadre général de l'étude

5 Au début du Ier siècle de notre ère, le sud-ouest de la péninsule coréenne connaît d'importants changements qui marquent le début du deuxième Âge du Fer ${ }^{2}$. On constate, entre autres, l'accroissement de la population et des villages, la multiplication des échanges à plus ou moins longue distance, la disparition des dolmens et le remplacement des armes et des outils en bronze par leurs homologues en fer qui commencent à être fabriqués localement. À la même période, le battage est progressivement utilisé pour la mise en forme et le décor des poteries. Du bassin du fleuve Han (région de l'actuelle ville de Séoul) jusqu'aux régions côtières de l'extrême sud-ouest, cette nouvelle production céramique coexiste pendant environ deux siècles avec celle de la période antérieure, dite de tradition mumun, qu'elle remplacera peu à 
peu. Parallèlement, l'évolution des techniques de cuisson des céramiques est marquée par l'apparition des premiers fours dits grimpants (tŭng yo en coréen), ancêtres des fours tunnels. Semi-excavés ou creusés en sape sur une pente, ils sont de forme ovale plus ou moins régulière. En moyenne, ils mesurent $6,3 \mathrm{~m}$ de long et présentent une inclinaison de 18,4 degrés. Dans la province du Chŏlla, les plus anciens sont datés du IIIe siècle, mais ils sont attestés dès le Ier siècle dans la province du Ch'ungch'ŏng, un peu plus au nord (Denès 2000 : 130-131).

6 Les céramiques à décor au battoir comprennent des terres cuites rouges, grises ou noires, ainsi que des poteries dures de couleur bleu-gris, parfois considérées comme des grès. Les pots globulaires ou ovoïdes, les jarres, les bols et les gobelets en constituent les formes les plus courantes, mais la production est peu standardisée. Les battoirs peuvent être entourés de cordes, mais ils sont plus souvent gravés de motifs de croisillons, de stries parallèles, ou de stries associées à un ou plusieurs traits perpendiculaires ou à un dessin en forme de patte d'oie. Sur le décor de surface obtenu avec ces battoirs s'ajoutent parfois des lignes horizontales incisées, et de larges zones peuvent également être lissées, notamment au niveau de l'épaule.

7 Selon l'opinion générale (Ch'oe Pyŏng-hyŏn 1994 : 123; Ch'oe Sŏng-nak 1993 : 146; Yi Yŏng-mun 1993 : 30; Barnes 1992 : 197; Yi Sŏng-ju 1991 : 235-238), ce nouveau type de céramique se serait diffusé et développé dans la péninsule coréenne en même temps que la culture de l'Âge du Fer, sous l'influence de la céramique chinoise grise à décor imprimé de l'époque des Royaumes Combattants (475-221 av. J.-C.) ou de la dynastie des Han (206 av. J.-C.-220 ap. J.-C.). Cependant, en l'état actuel des connaissances, on ne sait pas vraiment de quelle façon le battage s'est répandu en Corée, ni si les céramiques à décor au battoir du sud de la péninsule appartiennent à la même tradition que celles du nord qui sont attestées dès le IVe siècle av. J.-C.

8 Aux IIIe et IVe siècles de notre ère, les transformations socio-économiques liées à la généralisation de la métallurgie du fer et à la multiplication des échanges commerciaux aboutissent à la mise en place du royaume de Paekche dans le bassin du fleuve Han ${ }^{3}$. Cela favorise également le développement, dans l'extrême sud-ouest de la péninsule, d'une culture originale caractérisée par des sépultures en grandes jarres, relativement riches, enterrées au sommet de tertres funéraires (Denès 1995). Parallèlement, les céramiques à décor au battoir, devenues largement majoritaires, connaissent leur période d'apogée. Elles disparaîtront ensuite lentement, au cours des Ve-VIe siècles, remplacées par les céramiques grises à surface lissée, parfois décorées de motifs incisés, caractéristiques de la culture de Paekche qui s'étend peu à peu à l'ensemble du sudouest de la péninsule.

9 L'étude a porté sur deux cent douze céramiques de la période d'apogée (IIIe-IVe siècles), découvertes dans la province du Chŏlla Sud, dans l'extrême sud-ouest de la péninsule, et conservées dans les musées coréens. Ces vases -estimés représentatifs de la production commune de cette période par rapport à l'ensemble des céramiques observées dans les musées ou les dépôts de fouilles, ou encore décrites dans les diverses publications- ont été sélectionnés en fonction des critères suivants: forme archéologiquement complète, contexte de découverte connu et publié, descriptions suffisamment précises et illustrations de bonne qualité. Dix-sept vases sont toutefois moins bien connus. Ils proviennent en effet de fouilles très anciennes (notamment celles du tumulus $n^{\circ} 9$ de Sinch'on-ni) et ont été perdus ou mélangés. Ils ont néanmoins été inclus dans le corpus afin de ne pas dépareiller les ensembles clos (mobilier de 
tombes). Notons également que parmi les céramiques étudiées, cinquante-neuf (soit un peu plus d'un quart) ne présentent pas de traces de battage. Elles ont toutefois été conservées pour cette étude dans la mesure où elles appartiennent quand même d'après les archéologues coréens- à la catégorie des " céramiques à décor au battoir " en raison de similitudes de pâte et d'apparence générale. En outre, il n'est pas exclu qu'elles aient quand même été façonnées par battage, soit avec un battoir lisse, soit avec un battoir gravé dont les traces ont ensuite été lissées. Sont également rattachés à cette catégorie les bols très ouverts, les petits pots à grand col et les coupelles, mais ils ont été écartés du corpus car ces formes, probablement sans relation avec la technique du battage, n'apportaient rien au propos.

Les vases étudiés proviennent de trente tertres funéraires ( $71 \%$ des vases) et de trois zones d'habitat ( $29 \%$ des vases). Les tumuli renferment des structures variées : des cercueils en jarres, des sépultures en fosse avec ou sans entourage de pierres et une chambre funéraire en pierre. Les sites d'habitat, encore mal connus pour les IIIe-IVe siècles dans le sud-ouest de la péninsule, se caractérisent par des regroupements de fonds de cabane. Afin de simplifier cet exposé, les sites ont été rassemblés, en fonction de critères géographiques et chronologiques, au sein de trois groupes correspondant plus ou moins à trois étapes successives de la culture des sépultures en jarres (tab. 1, fig. 2). Tous les sites qui se trouvent en limite de la zone de répartition des sépultures en grandes jarres, supposés plus anciens que ceux du cœur de cette culture (région de l'embouchure du fleuve Yŏngsan), appartiennent au groupe I.

11 Il importe de remarquer que parallèlement à leur attribution chronologique et à leur nature (funéraire/domestique), les sites étudiés reflètent également d'autres différences. Ainsi, les sites d'habitats sont non seulement un peu plus anciens que l'ensemble des sites funéraires, mais ils se situent plus à l'est, le long du fleuve Posŏng et non dans la vallée du fleuve Yŏngsan (numéros 17-19, fig. 2). Des différences dans les techniques de fouilles et la conservation du matériel archéologique apparaissent aussi de façon très nette entre les sites du groupe III (postérieurs au milieu du IVe siècle), majoritairement fouillés entre 1917 et 1938, et ceux des groupes I et II (IIIe et début du IVe siècles), fouillés et étudiés surtout à partir des années 1980. Malgré les problèmes d'interprétation que ces différences peuvent entraîner, le choix a été fait de conserver l'ensemble des sites disponibles dans la mesure où, en 1996, au moment où j'ai mené mes recherches (examen des céramiques, collecte des rapports de fouilles), les sites retenus dans cette étude représentaient les données les plus fiables et les plus complètes pour la province du Chŏlla Sud. 
Figure 2. Carte de localisation des sites étudiés, province du Chŏlla Sud, Ille-IVe siècles

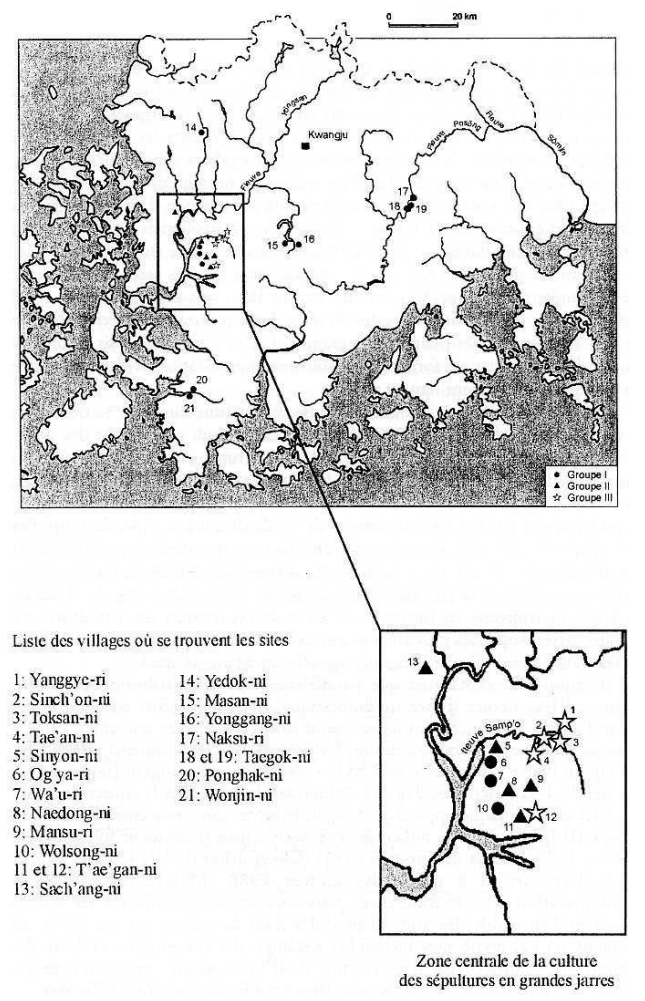

Le façonnage de la base des récipients

12 D'après les archéologues coréens qui se sont intéressés aux techniques de fabrication des céramiques protohistoriques du sud-ouest de la péninsule, celles-ci auraient été mises en forme grâce au battage à partir d'une ébauche montée aux colombins (Ch'oe Sŏng-nak 1993: 165-170; Sŏ Sŏng-hun \& Sŏng Nak-chun 1986:134-136). À ma connaissance, l'usage d'une autre technique pour la phase qui précède le battage (modelage ou montage par plaques, par exemple) ne semble pas avoir été envisagé.

13 L'usage du battage est attesté de façon claire et évidente sur la panse et la base des récipients (photo 1), non seulement par les traces laissées par le battoir décoré, mais également par les traces de contre-battoir présentes à l'intérieur de certains récipients. En revanche, la technique de façonnage de la partie supérieure des vases reste mal connue. Des traces de battage mal effacées sont visibles sur le col de nombreux vases, mais leur interprétation demeure incertaine. 
Photo 1. Céramique à décor au battoir $(\mathrm{H} .28 \mathrm{~cm})$.

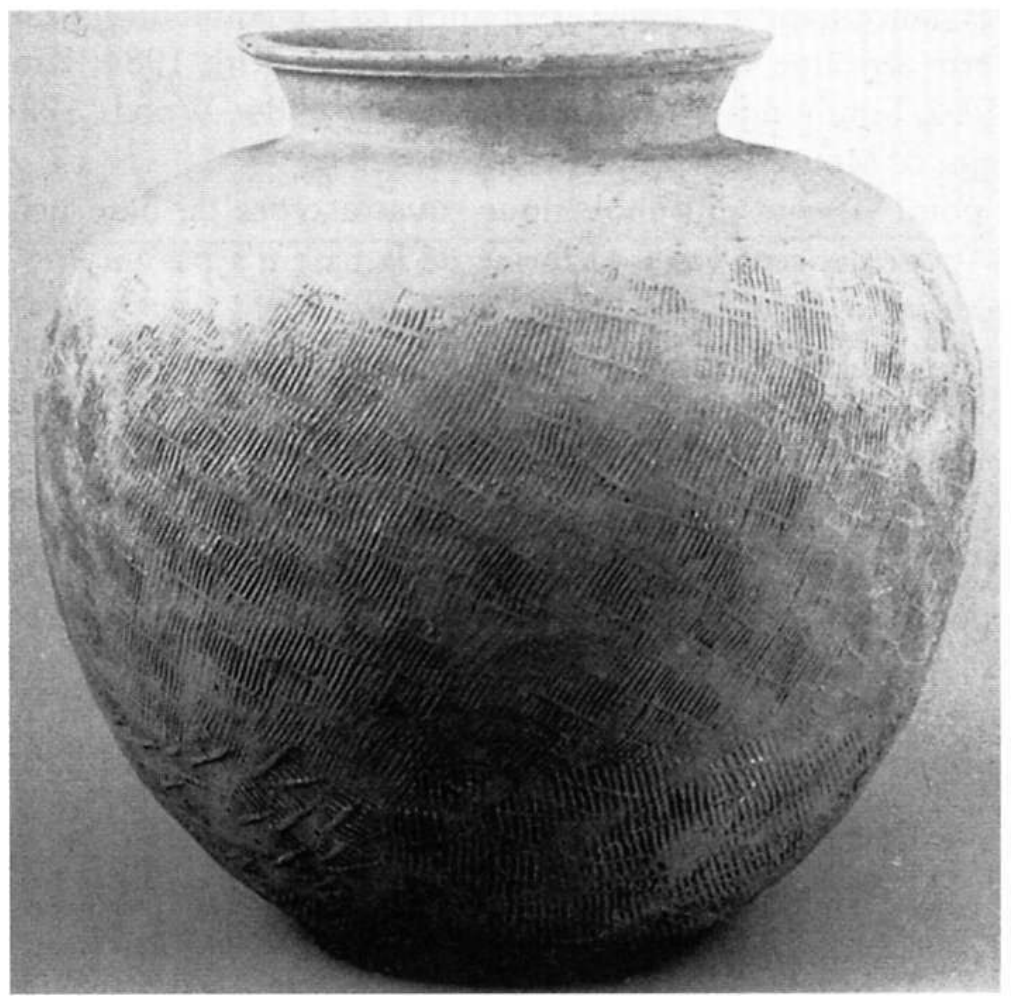

Traces de battage bien nettes sur la panse et la base, lissage sur l'épaule. Deux registres décoratifs distincts : sur la panse, décor régulier de stries parallèles recoupées par un trait perpendiculaire, et sur la base, décor plus grossier de stries parallèles recoupées par un motif en forme de patte d'oie. Site de Sinch'on-ni, tumulus $n^{\circ} 6$ (deuxième moitié du IVe - début du Ve siècles). D'après Sŏ Sŏng-hun \& Sŏng Nak-chun 1988 , p. $255, n^{\circ} 26$

Dans le cadre de ce travail, je me suis donc limitée aux différentes techniques de façonnage de la jonction entre la panse et la base des récipients, partie des céramiques intimement liée au battage. Il n'a pas été possible de réaliser des moulages des traces imprimées en surface par les coups de battoirs successifs, ni d'avoir recours à des analyses pétrographiques. Cependant, un certain nombre de traits propres aux effets décoratifs du battage ont pu être pris en compte, comme la distinction entre plusieurs types de décors au battoir sur un même vase, l'orientation et la qualité des motifs imprimés par la batte ou encore les relations entre battage et zones lissées. Différents éléments morpho-technologiques simples à observer ont aussi été retenus : un changement dans l'épaisseur de la paroi au niveau de la limite entre deux registres décoratifs, la présence de traces de lissage à la jonction entre la panse et la base, et la présence de fissures au niveau de cette même jonction. Les informations proviennent de mes observations ou des indications portées dans les rapports de fouilles. Je me suis également appuyée sur les études ethnographiques, nombreuses concernant le battage (Pétrequin \& Pétrequin 1999; Gallay et al. 1998; "Terre africaine » 1994; Ho Chuimei 1984; Kirk 1984; Kroun 1984; Mourer 1984; Simmonds 1984; van der Leeuw 1984; Woods 1984; Tobert 1984; Biagini \& Mourer 1971).

D'un point de vue morphologique, quatre types de base peuvent être distingués (pour dix-sept vases, la forme de la base n'a pas pu être identifiée avec certitude, $c f . p .1)$ :

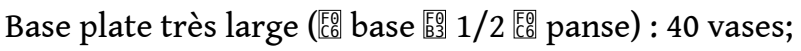

Base pointue ou arrondie : 22 vases; 
Base bien ronde ou légèrement aplatie : 84 vases;

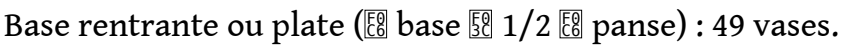
celles qui montrent un angle net par rapport à la panse (larges bases plates) et celles qui, au contraire, s'inscrivent dans le prolongement de la panse, sans angulation (bases rondes, pointues-arrondies et rentrantes).

Figure 3. Les différentes formes de bases des céramiques étudiées pour les Ille-IVe siècles (les décors éventuels ne sont pas indiqués)

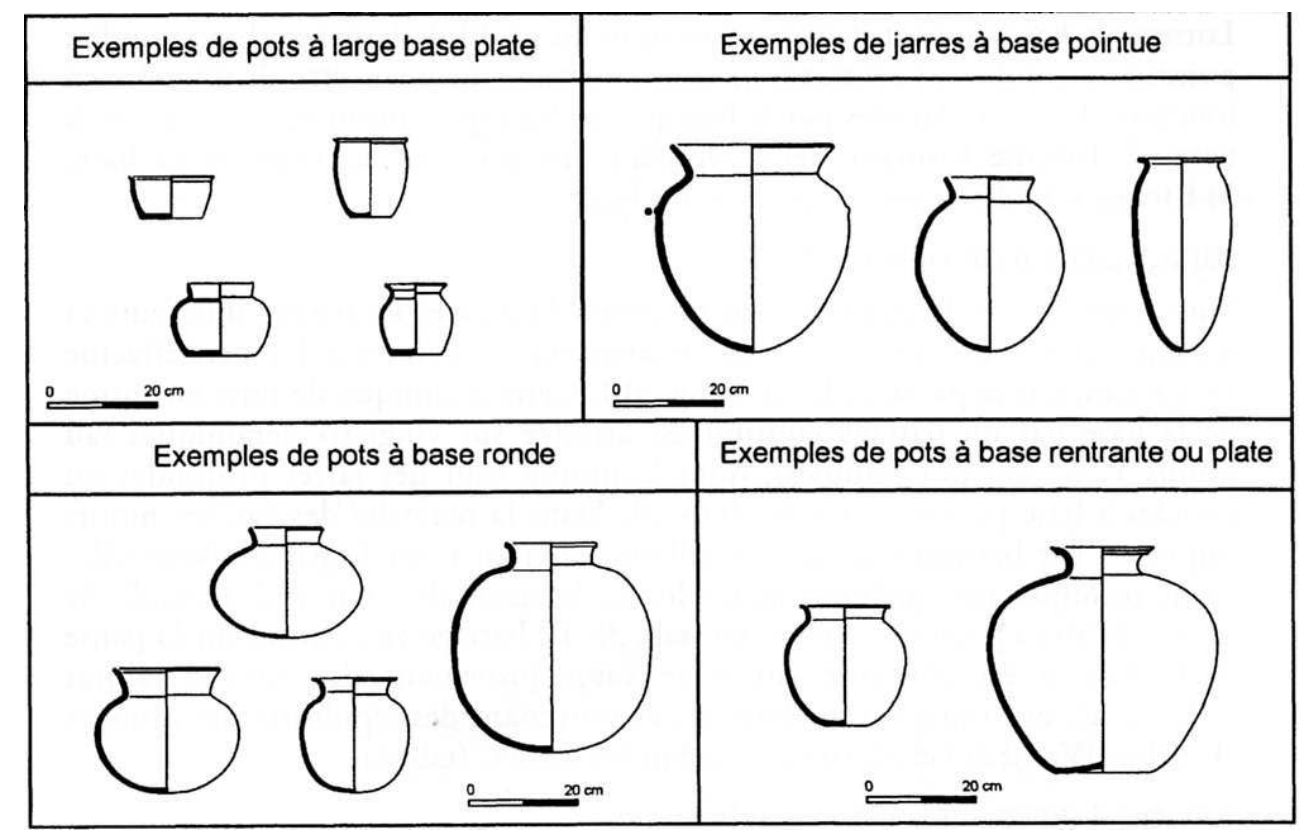

Angulation nette entre la base et la panse

Le corpus comprend quarante pots avec une large base plate, surtout fréquents dans les sites d'habitat du IIIe siècle (tab.4). Quatorze d'entre eux présentent des traces de battage sur la panse (surtout des motifs de croisillons, voir tab. 3) effacées par lissage au niveau de la jonction avec la base. Ces traces de lissage semblent indiquer que la panse a d'abord été façonnée comme un cylindre, légèrement mis en forme par battage, et que la jonction avec le disque de la base a été réalisée dans un second temps (fig. 4a). La seule analyse des profils de la paroi ne permet toutefois pas de le vérifier, mais ce procédé qui consiste à ajouter la base plate après façonnage de la panse est également décrit dans les études portant sur les céramiques de la période des Ier-IIIe siècles dans la même région (Ch'oe Sŏng-nak 1993: 177-179). De ce fait, on suppose, à titre d'hypothèse qu'il conviendra de vérifier ultérieurement, par des expérimentations 
notamment, que les vingt-six pots à large base plate sans trace de battage ont également été montés de cette façon.

Absence d'angulation nette entre la base et la panse

Lorsque la base s'inscrit dans la continuité du profil de la panse (bases rondes, pointues-arrondies et rentrantes), trois situations peuvent être distinguées en fonction des traces laissées par le battage : 1) battage continu sur la panse et la base; 2) battage formant deux registres distincts sur la panse et la base; 3) battage limité à la base, la panse étant lissée.

Battage continu sur la panse et la base

21 Sur certains vases, le décor au battoir couvre la base et les parties inférieure et médiane de la panse en un seul registre uniforme. Le battage a donc été effectué en continu sur la panse et la base (fig. 4b). Cette technique de mise en forme de la base par un battage continu est attestée sur vingt-six céramiques (au moins $12 \%$ des pots étudiés), dont la moitié sont des jarres profondes ou ovoïdes à base pointue-arrondie (tab. 2). Dans la majorité des cas, les motifs imprimés par battage sont des croisillons couvrant toute la panse, éventuellement recoupés par quelques lignes lissées horizontales. Un seul exemple de motifs de stries parallèles a été noté (tab.3). Le battage en continu sur la panse et la base a été identifié sur seize vases provenant des sites d'habitat (IIIe siècle), ainsi que sur quelques vases provenant des sépultures des groupes II (début IVe siècle) et III (mi-IVe-début Ve siècles) (tab. 4).

Battages distincts sur la panse et la base

Une autre technique de façonnage de la base a été identifiée sur soixante-treize céramiques (au moins $34 \%$ des pots étudiés, tab. 2) qui présentent deux registres distincts (photo 1) sur la panse et la base (deux décors au battoir différents par leurs motifs ou par l'orientation des traces de battage). Un montage en deux phases successives peut ainsi être mis en évidence (fig. 4c). Dans $40 \%$ des cas (vingt-neuf individus) la limite entre les deux registres correspond à un changement dans l'épaisseur de la paroi, à une fissure et/ou à des traces de lissage.

D'autre part, sur au moins treize vases, les traces du battage de la base recoupaient celles de la panse, indiquant ainsi l'ordre de succession des opérations réalisées. Sur deux de ces pièces, le décor au battoir de la base recouvrait aussi de façon très nette les lignes horizontales incisées sur le décor au battoir de la panse. L'ensemble du décor de la panse aurait donc été réalisé avant le façonnage définitif de la base.

La plupart des vases sur lesquels on reconnait ce processus de façonnage en deux étapes distinctes possèdent une base ronde, aplatie ou rentrante, plus rarement pointue (tab. 2). Contrairement au cas du battage continu sur la base et la panse, les motifs de stries parallèles sont ici largement majoritaires sur la panse et la plupart des motifs de croisillons sont associés à une large zone lissée sur l'épaule. Les motifs cordés sont également bien représentés (tab. 3). Ces vases proviennent de l'ensemble des sites étudiés (tab. 4). 
Figure 4. Représentations schématiques des différentes techniques de battage de la panse et de la base des vases. La forme de l'ébauche est seulement donnée à titre indicatif, la technique d'ébauchage n'ayant pas fait l'objet d'une étude à ce stade

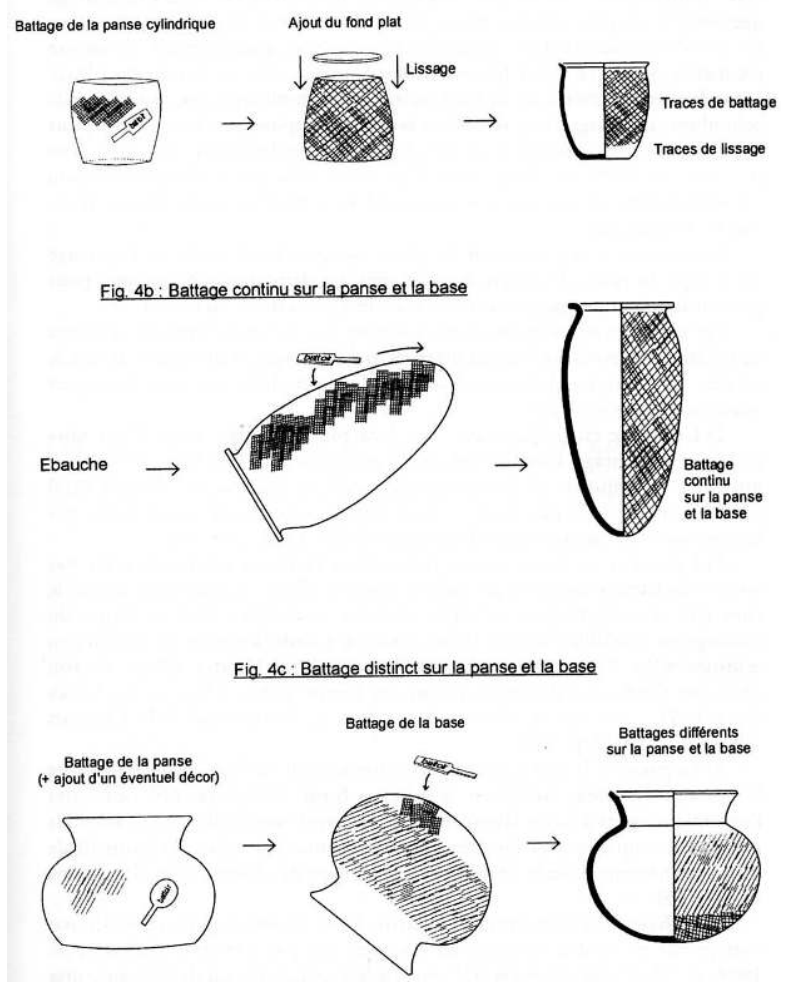

Battage sur la base, panse lissée

Seize individus (7,5\% des pots étudiés) ne présentent des traces de battage que sur la base, généralement ronde (tab. 2). Les motifs les plus fréquents sont les croisillons (tab. 3). Dans le cas de ces récipients, aucune étude n'a encore été menée pour savoir si la panse a d'abord été travaillée au battoir puis lissée avant la mise en forme de la base ou si elle a simplement été façonnée aux colombins, le battage étant réservée à la base. L'emploi d'un battoir lisse pour la panse n'est pas non plus à exclure. Comme précédemment, plus d'un tiers des vases comporte un changement d'épaisseur de la paroi, des fissures et/ou traces de lissage au niveau de la jonction base/panse (six individus sur seize, soit $37,5 \%$ des cas).

Il serait tout à fait instructif de mieux comprendre le mode de façonnage de ce type de vases, découvert dans les sites des deux premiers groupes, pour pouvoir les situer par rapport aux autres céramiques à décor au battoir.

Les exemples ethnographiques montrent que les trois types de relations base/panse observées au sein du matériel archéologique étudié peuvent être le résultat de multiples chaînes opératoires. Pour simplifier, on peut distinguer quatre tendances générales.

1) L'ébauche est préparée avec une base ronde ou plate avant d'être mise en forme par battage. Cette technique est pratiquée dans l'île Yami, à l'extrême nord des Philippines : le potier travaille d'abord des plaques d'argile qu'il assemble en une sorte de cylindre à fond plat qui sera ensuite mis en forme par battage pour obtenir une jarre à fond rond (Kroun 1984 : 287-291). 
29 2) L'ébauche se limite à une panse dont la partie inférieure n'est pas fermée : le battage permet d'arrondir la panse et d'étirer la pâte pour fermer la base. On trouve plusieurs exemples de cette technique : dans la région du Kavango en Namibie (Woods 1984 : 306); au Cambodge pour les céramiques traditionnelles (Biagini \& Mourer 1971 : 201-206; Mourer 1984 : 29-30); dans l'île Negro aux Philippines (van der Leeuw 1984 : 334); au Sri Lanka (Kirk 1984 : 2-3); ou encore dans le nord-est de la Thaillande (Ho Chuimei $1984: 263-264$, ill. p. 278).

30 3) La panse et la base sont préparées séparément : le battage assure ensuite la jonction des deux parties et la mise en forme définitive. On peut citer l'exemple de pots à cuire fabriqués sur l'île Negro aux Philippines : les deux parties des récipients sont moulées, puis jointes une fois sèches, la forme finale étant donnée par battage après une autre phase de séchage (van der Leeuw 1984 : 335).

31 4) La base peut être façonnée à partir d'une plaque d'argile travaillée par battage sur un moule convexe, par exemple un pot retourné (Gallay et al. 1998, pl. 16-17, fig. 79, 82 et 84). Au Nigeria (village Hausa de Naraguta près de Jos), la base est moulée à partir d'une plaque d'argile circulaire placée sur le fond d'un pot retourné et martelée avec une batte en bois jusqu'à obtention d'une forme semi-sphérique. La pièce est ensuite retravaillée par battage et complétée aux colombins (Simmonds 1984 : 58). On trouve aussi une méthode proche, pour des formes rondes à large ouverture, chez les potières Bamana de Kalabougou au Mali (« Terre Africaine » 1994 : 32).

Dans le cas d'une base moulée, la panse est généralement ajoutée ensuite. Dans les trois premiers cas, la mise en forme finale par battage peut s'effectuer soit en continu sur la panse et la base, soit de façon distincte pour la base, en retournant la pièce et/ou en changeant d'outil entre les deux étapes (battage de la panse/battage de la base).

En définitive, il est difficile, avec les données aujourd'hui disponibles, de déterminer le type d'ébauche réalisé avant battage. La solution serait sans doute de mettre en œuvre un programme d'expérimentations afin de tester les différentes chaînes opératoires possibles et de comparer les vases expérimentaux aux vases archéologiques. Il serait notamment intéressant de savoir si la dernière méthode (battage de la base sur un moule convexe) n'a pas pu être employée pour les céramiques du corpus dont seule la base est travaillée par battage.

L'évolution du battage et des formes de céramiquesIer-IIe siècles : apparition et développement du battage

34 À la période où apparaissent les premières céramiques à décor au battoir, la production dominante est caractérisée par des pots et des jarres sans décor, montés aux colombins, dont toutes les formes présentent une base plate. Leur ouverture est généralement évasée (figure 5A, B) et certains récipients sont munis de préhensions en forme de corne de bœuf. Cette production, relativement homogène, dite de tradition mumun ("sans décor» en coréen), se situe dans la continuité des céramiques de l'Âge du Bronze (Ch'oe Sŏng-nak 1993 : 142, 144).

Les plus anciennes céramiques avec des traces de battage (généralement des motifs de croisillons sur toute la panse) restent assez mal connues. Il semble que d'un point de vue morphologique elles se situent dans la continuité des céramiques de tradition mumun, avec cependant l'apparition, notoire, de jarres avec une base ronde. Ces nouvelles formes, instables sans support, impliquent certainement des changements dans l'utilisation des récipients, mais les données archéologiques liées au contexte de 
découverte des céramiques n'apportent pas d'informations à ce sujet. Curieusement, cette importante évolution morphologique ne ressort pas des études de M. Ch'oe Sŏngnak, pourtant très précises et approfondies pour les trois premiers siècles de notre ère dans le sud-ouest de la péninsule. De son point de vue, la plupart des formes des céramiques à décor au battoir sont héritées de celles de tradition mumun, l'apparition des pots à panse et base rondes n'étant indiquée que de façon presque anecdotique (Ch'oe Sŏng-nak 1993: 163). Peut-être faut-il y voir le reflet du peu d'intérêt généralement porté au battage et à ses caractéristiques. Dans tous les cas, on connaît mal les modalités d'apparition et de développement des premières céramiques à base ronde.

IIIe siècle-première moitié du IVe siècle : apogée du battage

Parmi les céramiques du corpus étudié, certaines s'inscrivent parfaitement dans la continuité de la production des deux premiers siècles de notre ère (fig. $5 \mathrm{C}$ ).

Il s'agit tout d'abord des pots à large base plate et à panse très peu bombée, dont un peu plus d'un tiers présente des traces de battage, seule différence notable par rapport aux pots de tradition mumun. Ces récipients, surtout fréquents dans les sites du IIIe siècle, se raréfient ensuite progressivement (tab. 5).

Les jarres sur lesquelles le battage de la panse et de la base est uniforme se situent également dans la continuité des plus anciennes céramiques décorées par battage connues pour le sud-ouest de la péninsule. Comme nous l'avons vu, elles se distinguent cependant, par leur base arrondie, des jarres de tradition mumun dont l'assise, même très étroite, est toujours plate (fig.5). Elles proviennent principalement des sites d'habitat du IIIe siècle, mais on les trouve aussi dans les sépultures du IVe siècle (tab. 4 et 5). Celles qui ont été découvertes dans les fonds de cabane sont très profondes ou ovales, à fond presque pointu et ouverture très simple (H entre 30 et $40 \mathrm{~cm}$ environ). On suppose qu'elles ont servi à faire bouillir de l'eau du fait de leur fréquente association avec des vases dont le fond est percé de plusieurs trous, probablement pour la cuisson à la vapeur. Elles sont parfois aussi utilisées pour former des cercueils en jarre(s) de petite taille. (Voir par exemple les sépultures en jarre(s) $n^{\circ} 6$ et $n^{\circ} 15$ du tumulus $n^{\circ} 4$ de Mansu-ri à Yŏngam. Sŏng Nak-chun \& Kim Kil-sik 1990 : 59).

En revanche, les céramiques dont la base a été façonnée selon un processus distinct de celui de la panse, ainsi que celles dont seule la base est travaillée par battage, ne trouvent pas d'échos, du point de vue des formes, des décors ou des techniques de façonnage, dans les céramiques des premiers siècles de notre ère connues pour la région du Chŏlla Sud. Les premières représentent pourtant déjà environ un tiers de la production dans les sites du IIIe siècle. Elles deviennent ensuite largement majoritaires (tab. 5). Il serait intéressant d'approfondir la question de leur origine. S'agit-il d'une évolution locale au cours du IIIe siècle? Ou d'un apport extérieur, peut-être depuis la région du bassin du fleuve Kŭm plus au nord? 
Figure 5. Évolution des formes entre le ler siècle avant J.-C. et le milieu du Ille siècle de notre ère dans la province du Chŏlla Sud.

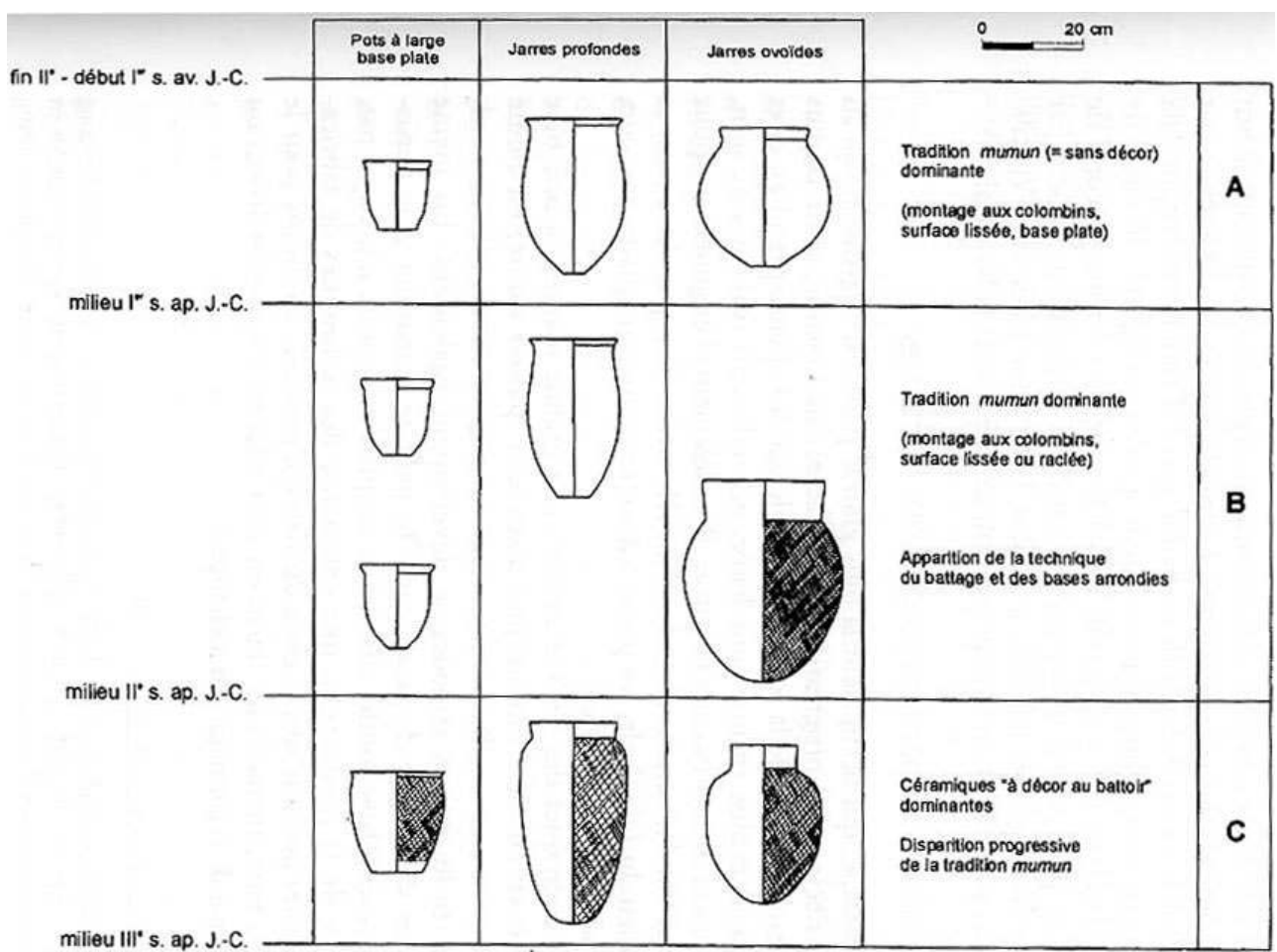

Ce tableau s'inspire en partie de celui de Ch'oe Sŏng-nak 1993 (tableau n² 2, p. 243), mais les vases ont été remis à l'échelle. À partir du Ille siècle, de nouvelles formes apparaissent et la production devient très hétérogène, rendant difficile le choix de récipients représentatifs

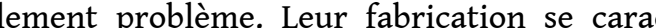
entouré de cordes, et non gravé. Dans notre corpus, dix vases relativement homogènes appartiennent à cette catégorie. Il s'agit de grands pots globulaires ( $30 \mathrm{à} 40 \mathrm{~cm}$ de haut) à ouverture étroite, décorés de façon tout à fait particulière d'impressions cordées verticales recoupées par plusieurs lignes horizontales incisées, avec une large zone lissée sur l'épaule. Deux d'entre eux présentent un battage continu sur la panse et la base (tab. 3).

Milieu du IVe siècle : disparition progressive du battage

Les traces du battage, qui occupaient la plus grande partie de la panse jusqu'au début du IVe siècle, sont progressivement effacées sur l'épaule, zone la plus visible des vases. Le lissage de la jonction entre le col et la panse prend en effet de l'importance et évolue en une zone lissée, généralement réalisée avec soin, qui s'étend plus ou moins bas sur la panse. Parallèlement, l'engouement pour les formes à base ronde, surtout abondants au IIIe siècle, finit par s'atténuer, et à partir du milieu du IVe siècle, les pièces à base rentrante ou aplatie, toujours façonnées au battoir, se multiplient. 

qui doit être travaillée lorsqu'elle a la consistance du cuir. Si l'argile est trop molle, l'outil sera vite obstrué; si au contraire elle est trop sèche, le battage n'aura que peu d'effets et pourra provoquer des fissures (Pétrequin \& Pétrequin 1999: 75; Gibson \& Woods 1990 : 42-43). En revanche, les avantages ne manquent pas. Cette technique, qui permet d'étendre la paroi et de la rendre très fine, réduit en effet la porosité de la pâte en augmentant sa cohésion, sa densité et sa solidité (Pétrequin \& Pétrequin 1999 : 75; Gibson \& Woods 1990 : 95-96; Mourer $1984: 31$ ). Notons que dans les exemples khmers, pour ne citer que ceux-là, un grand vase de $45 \mathrm{~cm}$ de diamètre fabriqué par battage présente une paroi d'environ $3 \mathrm{~mm}$, pèse moins de $3 \mathrm{~kg}$ et peut porter un poids qui correspond à sa capacité de 20 à 30 litres (Mourer 1984:32). Pour ces différentes raisons, les pots travaillés par battage sont employés pour le transport et la conservation des liquides, aussi bien que pour la cuisson des aliments (Gibson \& Woods 1990 : 95-96; Tobert $1984: 223$; Simmonds $1984: 57,63$; Kroun $1984: 286$ ). C'est en outre une technique fiable et économique (Kirk $1984: 3$ ), qui ne nécessite qu'un matériel très simple (Simmonds $1984: 58$ ). alors que ceux qui se trouvent à la base des pots renforcent leur solidité et leur alors que ceux qui se trouvent à la base des pots renforcent leur solidité et leur procurent une assise plus stable grâce à la rugosité du relief (Mourer 1984 : 33; Biagini \& Mourer 1971: 207-208, fig. 18-19 p. 214 et fig. 20 p. 215). La rugosité de la surface obtenue par martèlement avec un battoir non lisse pourrait également accroître la zone de surface et favoriserait l'absorption rapide de la chaleur du feu ${ }^{5}$ (Simmonds $1984: 64)$.

D'autre part, les céramiques à base ronde et corps globuleux s'avèrent bien adaptées à la cuisson des aliments, car leur forme supporte mieux les déformations dues aux 
chauffages et refroidissements successifs. Dans le cas des fonds plats, l'angle situé à la jonction entre la base et la panse risque de casser lors de ces déformations (Gibson \& Woods 1990 : 34). Les vases à fond rond sont en outre facilement placés sur un foyer constitué de quelques pierres (Mourer 1984: 33), sur lequel ils peuvent être tournés régulièrement pour obtenir une meilleure répartition de la chaleur et donc une cuisson plus homogène des aliments. Placés sur un anneau, ils sont également très pratiques pour un transport sur la tête. Lorsque la base est plus pointue (comme pour les jarres profondes) ils sont bien adaptés pour être portés sur l'épaule avec les bras levés, plutôt que sur la tête (Simmonds 1984 : 63-64). Grâce à leur forme, ils peuvent également être placés dans un trou creusé dans le sol pour servir au stockage à long terme.

Le battage et les nouveaux types de four

Dans le sud-ouest de la péninsule coréenne, les céramiques des IIIe-IVe siècles présentent souvent des traces "d'accidents »; elles constituent donc de bons témoins des difficultés rencontrées par les potiers. Avant d'analyser la nature de ces « accidents » et de voir ce qu'ils révèlent sur la maîtrise des techniques et leur relation avec le battage, disons quelques mots de l'argile et des modes de cuisson.

Le choix de l'argile et sa préparation ne sont pas sans conséquences, non seulement pour obtenir de bons résultats en fonction de la technique de montage choisie, mais surtout pour limiter les risques d'accidents (déformations, fissures, éclatements) lors des phases de séchage et de cuisson. Pour ne donner qu'un exemple, on note, dans une étude ethnographique réalisée au Soudan, l'emploi de deux dégraissants différents pour la fabrication d'un même vase, l'un pour le corps qui est travaillé par battage, l'autre pour le col et la lèvre qui sont montés aux colombins (Tobert 1984 : 223). La fonction de la pièce peut également entrer en ligne de compte, une argile grossière étant mieux adaptée, par exemple, à des récipients destinés à la cuisson des aliments (Simmonds 1984 : 65). Le battage, quant à lui, nécessite une argile ferme, solide et assez plastique, mais pas trop collante, c'est-à-dire bien dégraissée, assez rugueuse (Mourer $1984: 31)$.

51 Dans les rapports de fouilles coréens, les indications disponibles sur l'argile concernent le plus souvent l'aspect général de la pâte (fine ou grossière), la nature et l'importance des éléments non plastiques (argiles ne contenant pratiquement pas d'inclusions, argiles peu ou très sableuses, argiles contenant des inclusions autres que sableuses), ainsi que la couleur et le degré de dureté des pâtes, ces deux dernières caractéristiques étant liées non seulement au type d'argile employé, mais aussi au degré et au mode de cuisson. Toutefois, ces données ne sont pas toujours très précises et homogènes, et il est pour l'instant difficile d'en tenir compte pour aborder le problème de la maitrise des techniques, notamment des relations entre l'argile, les techniques de montage et la cuisson.

Certains archéologues coréens (Ch'oe Pyŏng-hyŏn 1994 : 123; Yi Yŏng-mun 1993 : 30-31) considèrent que les premiers fours grimpants (proto-fours tunnels) sont apparus dans le sud-ouest de la péninsule à peu près en même temps que le battage, aux environs du premier siècle de notre ère. Cependant, en l'état actuel des connaissances, on ne peut dire s'il s'agit d'une coïncidence ou si les deux techniques ont une origine commune. De plus, on ne sait pas selon quelles modalités ces nouvelles techniques se sont diffusées. En fait, les plus anciens fours grimpants connus dans le sud-ouest de la péninsule sont datés entre le début du Ier et la fin du IVe siècle dans la région du fleuve Kŭm et du IIIe siècle dans la région étudiée ici, située un peu plus au sud. On y cuisait 
vraisemblablement à la fois des céramiques de tradition mumun et des céramiques à décor au battoir (Barnes 1992 : 205-206).

Sur trente-cinq pièces du corpus, on observe de larges taches noires de formes irrégulières, correspondant à des dépôts de carbone dus au contact direct avec les flammes et les fumées ou avec du combustible partiellement consumé. Ces traces de coups de feu sont généralement liées à une cuisson en foyer ouvert, mais les changements de couleur localisés peuvent se produire dans n'importe quel type de four. En outre, ces traces peuvent être dues à une utilisation ultérieure des récipients pour cuire les aliments (Orton et al. 1993 : 222-223; Gibson \& Woods $1990:$ 47-49, 106). $\mathrm{Au}$ moins deux vases présentent sur l'extérieur de la base des traces rectangulaires imprimées assez nettement qui témoignent peut-être de l'usage de matériel d'enfournement. Il est donc malaisé de définir le type de cuisson qui correspond à chaque pièce et de savoir si elles ont été cuites dans les fours grimpants ou dans des fours plus rudimentaires.

Certains éléments s'avèrent néanmoins intéressants dans la mesure où ils témoignent des difficultés rencontrées par les potiers dans la maîtrise technique de la fabrication des céramiques, même si, dans certains cas, la possible recherche d'un aspect décoratif ne peut être écartée ${ }^{6}$ (tab. 6).

Les déformations constituent les témoins les plus impressionnants et les plus significatifs. Sans contour précis, elles affectent toute la céramique ou seulement une partie, souvent l'ouverture. Elles sont présentes sur quarante-deux vases du corpus (près de $20 \%$ de l'ensemble), dix étant complètement déformés. La production d'un potier comprend toujours des pièces plus ou moins bien réussies, mais les céramiques déformées sont ici particulièrement nombreuses, et surtout, elles sont utilisées, et même placées dans des sépultures riches, et non pas abandonnées près des fours. Pour ne donner que deux exemples, neuf céramiques présentant des déformations étaient associées aux sépultures du tumulus $n^{\circ} 9$ de Sinch'on-ni (dont trois pour la sépulture contenant une couronne et des souliers en bronze doré) et cinq à celles du tumulus $n^{\circ} 9$ de Tae'an-ni, deux tumuli qui sont parmi les plus riches de la région à cette période.

Les déformations des vases peuvent être attribuées à trois causes principales: le manque de connaissance du matériau; l'incapacité de travailler ce matériau; une mauvaise compréhension du processus de cuisson du four (Mourer 1984 : 33; Gibson \& Woods $1990: 24,151$; Sanders \& Tomimoto $1976: 115-116)$. Les déformations et autres problèmes rencontrés par les potiers résultent donc souvent d'une mauvaise adéquation entre l'argile, les techniques de montage et la cuisson. Notamment, lorsque l'argile contient trop de fondants (surtout des oxydes métalliques), comme cela semble être le cas dans le sud de la péninsule coréenne (Tite et al. 1992: 66-68; Barnes 1992 : 200), elle fond et se déforme lors d'une cuisson à température élevée (ou irrégulière), sauf si le potier ajoute du sable ou un autre dégraissant pour la rendre moins fusible (Mourer 1984 : 31; Sanders \& Tomimoto 1976 : 115-116; Biagini \& Mourer 1971 : 205-206, 212-213, 219). Dans ce cas, les déformations sont associées à des cloques ou à des traces de couverte accidentelle. Les cloques (bien nettes sur huit individus de notre corpus) sont des poches d'air, intactes ou éclatées, qui boursouflent, de façon parfois spectaculaire, la surface des vases et peuvent aller jusqu'à en déformer complètement la paroi (photo 2). Elles sont causées par l'évolution des gaz pendant la cuisson, surtout quand celle-ci a été trop rapide et quand la première étape de la vitrification s'est produite avant que les matières organiques ne soient complètement brûlées (Gibson \& 
Woods 1990 : 106). Les traces de couverte accidentelle (appelée « couverte naturelle » en coréen) ont été relevées sur six vases de notre corpus, le plus souvent sur l'épaule. La formation de cette couverte peut être due à une vitrification partielle de la pâte, notamment lorsque celle-ci est riche en fondants, mais certains auteurs attribuent également ce phénomène à des retombées de cendres chaudes à haute température. Sanders et Tomimoto (1976: 28 et fig. 6, p. 28 ) parlent dans ce cas de "glaçure » accidentelle.

Les renfoncements correspondent à des déformations plus ou moins circulaires et relativement localisées. Il peut y en avoir plusieurs sur une même pièce, et vingt-trois vases (près de $11 \%$ de l'ensemble du corpus) en présen-tent au moins un. La cause de ce type de déformation serait la position de la céramique lors de la cuisson, probablement allongée sur le côté et calée avec des pierres (Sŏng Nak-chun \& Kim Kil-sik 1990 : 65), ou placée en contact avec d'autres poteries pendant les phases de séchage ou de cuisson. Sur quatre pièces, on note en effet la présence d'un morceau d'une autre céramique resté collé au niveau du renfoncement, ce qui tend à montrer que les pots pouvaient se toucher lors de la cuisson, voire éventuellement s'affaisser les uns sur les autres.

Photo 2. Céramique à décor au battoir $(\mathrm{H} .38,5 \mathrm{~cm})$

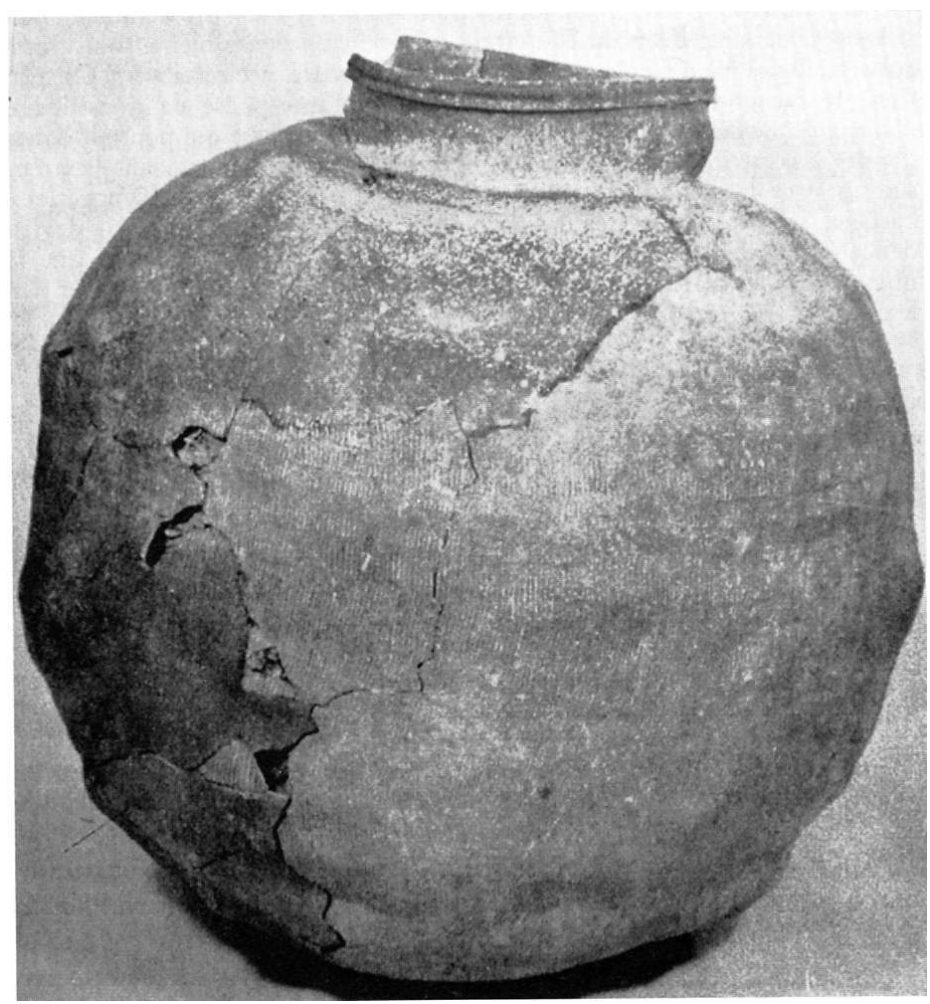

Déformations avec cloques dues à une mauvaise adéquation entre l'argile utilisée et la technique de cuisson. Battage cordé sur la panse et la base, recoupé par des lignes horizontales incisées sur le milieu de la panse. Large zone lissée sur l'épaule. Site de Naedong-ni Ch'obun'gol, tumulus n 2 (première moitié ou milieu du IVe siècle). D'après Sŏ Sŏng-hun \& Sŏng Nak-chun 1986, p. 189, nº 77

Les taches circulaires (huit individus dans notre corpus) constituent d'autres éléments qui reflètent les problèmes rencontrés par les potiers. Ces taches, situées sur la panse ou l'épaule, se caractérisent par leur forme bien ronde (photo 3). Elles sont d'une couleur différente de la couleur principale du vase, mais pas nécessairement noires, et pourraient résulter de la présence d'un support ou du contact avec l'ouverture d'un 
autre vase lors de la cuisson. Elles sont associées à des renfoncements dans six cas sur huit.

Ces « accidents" (déformations, boursouflures, etc.) proviennent essentiellement des sites datés du IVe ou du début du Ve siècle, même si on trouve aussi quelques exemples de déformations dès le IIIe siècle (tab. 6). On peut donc se demander s'ils ne sont pas le reflet d'une phase de transition et d'adaptation à laquelle ont été confrontés les potiers, phase qui pourrait correspondre à la période d'adoption des premiers fours grimpants dans l'extrême sud-ouest de la péninsule. Si l'on considère qu'à cette période (IVe siècle) le battage, très largement répandu, semble parfaitement maîtrisé, il devient délicat de soutenir l'hypothèse qu'il soit apparu en même temps que les nouveaux types de fours dans le sud-ouest de la péninsule, en particulier dans le cas de la province du Chŏlla Sud.

Photo 3. Céramique à décor au battoir (H $29 \mathrm{~cm})$.

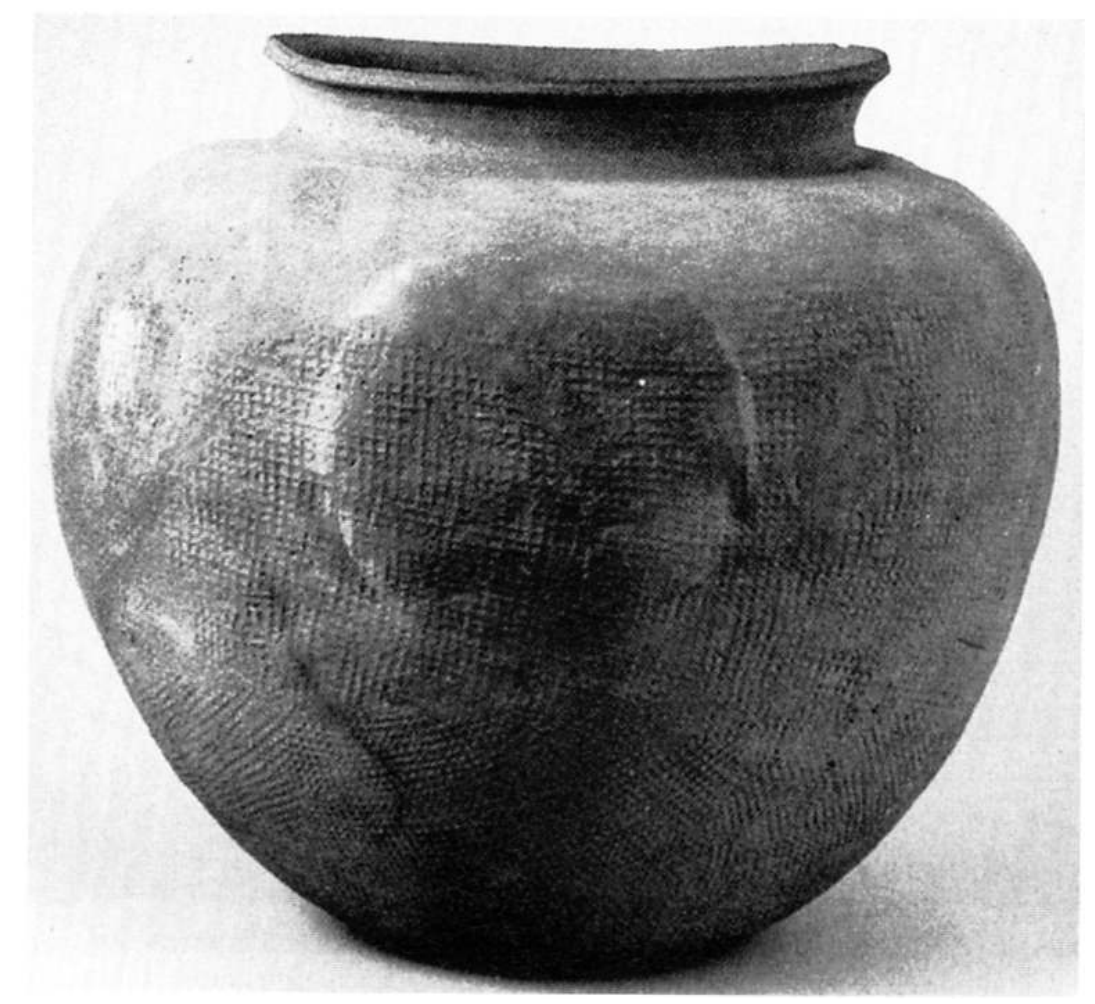

Large tache noire circulaire sur la panse. Décors de croisillons formant deux registres décoratifs distincts sur la panse et la base, lissage sur l'épaule. Site de Tŏksan-ni, tumulus $n^{\circ} 3$ (deuxième moitié du IVe - début du Ve siècle). D'après Sŏ Sŏng-hun \& Sŏng Nak-chun 1988, p. 250, n²0-2

L'étude menée sur les formes, les décors et les techniques de fabrication des céramiques à décor au battoir des IIIe-IVe siècles dans la province du Chŏlla Sud permet de suggérer l'existence de plusieurs traditions différentes. L'une de ces traditions reflète une évolution locale et continue des céramiques de la fin de l'Âge du Bronze, d'abord sensible dans la forme des vases (pots à large fond plat, jarres profondes ou ovoïdes dont la base s'arrondit), avec l'emploi d'un battoir gravé de motifs de croisillons. En revanche, on ne trouve pas trace des autres traditions céramiques dans la région avant le IIIe siècle. L'usage de battoirs gravés de stries parallèles associé à une technique de façonnage plus complexe, la base des récipients étant travaillée par battage selon un processus distinct de celui de la panse, pourrait 
avoir une origine extérieure à la province du Chŏlla. Cette origine serait peut-être à rechercher dans le bassin du fleuve Kŭm, un peu plus au nord. L'utilisation d'un battoir cordé, et non gravé, pour une partie de la production pose également problème. Les vases dont seule la base présente des traces de battage, la panse étant lissée, constituent encore une autre tradition dont la valeur culturelle ou chronologique reste mal établie. Sa localisation parait limitée, mais les données sont encore insuffisantes pour en tirer des conclusions. Il serait notamment intéressant de connaitre les techniques de façonnage de ces vases (battage soigneusement lissé, battoir lisse ou colombins sans battage). Les vases qui n'ont conservé aucune trace de battage, mais dont la technique de fabrication n'a pas été examinée, renvoient à un autre aspect problématique de la production céramique.

61 En définitive, il conviendrait d'une part d'élargir le corpus d'étude géographiquement, notamment vers le nord, et d'autre part de pouvoir approfondir la connaissance des techniques de fabrication en ayant recours, entre autres, à des observations pétrographiques. On comprendrait mieux ainsi le processus complexe, et probablement non linéaire, de développement des différentes traditions liées à la technique du battage dans la province du Chŏlla. Il semble en effet que seule une approche régionale fine permette, à plus ou moins long terme, d'appréhender les modalités d'une éventuelle influence des céramiques chinoises.

Tableau 1. Sites étudiés, classés par ordre chronologique

\begin{tabular}{|c|c|c|c|c|c|}
\hline $\begin{array}{l}\text { Groupes } \\
\text { de sites }\end{array}$ & $\begin{array}{l}\text { Datation } \\
\text { proposée }\end{array}$ & Sites & Types de sinucture & $\begin{array}{l}\text { Année de } \\
\text { fouilles }\end{array}$ & $\begin{array}{l}\mathrm{N}^{\circ} \text { sur } \\
\text { la carte }\end{array}$ \\
\hline \multirow{12}{*}{1} & \multirow[b]{2}{*}{ IJIs siecle } & $\begin{array}{l}\text { Naksuri Naksu, } \\
\text { zone d'habilat }\end{array}$ & $\begin{array}{l}15 \text { fonds de cabane } \\
\text { (3 ont ééé retenus ici) }\end{array}$ & | 1986-1987 & 17 \\
\hline & & $\begin{array}{l}\text { Taegok-ni, Torong, } \\
\text { zone d'liabilat }\end{array}$ & $\begin{array}{l}\text { Nombreux fonds de cabanc } \\
\text { (16 ont été relenus ici) }\end{array}$ & $\begin{array}{l}1986, \\
1987, \\
1989 \\
\end{array}$ & 18 \\
\hline & $\begin{array}{l}\text { III ou } \\
\text { IN siecle }\end{array}$ & $\begin{array}{l}\text { Taegok-ni, Hansil, } \\
\text { zone d'habital }\end{array}$ & $\begin{array}{l}9 \text { fonds de cabane } \\
\text { (1 seul a ććć relenu ici) }\end{array}$ & 1989 & 19 \\
\hline & \multirow{5}{*}{ III' siecle } & $\begin{array}{l}\text { Yedök-ni, Man' garch'on, } \\
\text { tumulus } \mathrm{n}^{\circ} 6\end{array}$ & $\begin{array}{l}\text { Sépultures de type de inconnu, } \\
\text { supposés en jares. }\end{array}$ & sondages & 14 \\
\hline & & $\begin{array}{l}\text { Yonggang-ni, } \\
4 \text { tumuli supposés }\end{array}$ & 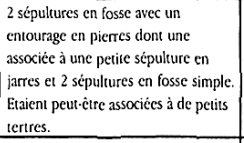 & $\begin{array}{l}1989 \\
1996\end{array}$ & 16 \\
\hline & & $\begin{array}{l}\text { Masann-nil, Chaenggi } \\
\text { mơrti, } \\
\text { tumulus supposé }\end{array}$ & $\begin{array}{l}3 \text { sépultures en fosse avec un } \\
\text { entourage en pierres et une } \\
\text { sépulture en grandes jarres. Peutüture } \\
\text { dans un méme cersre. }\end{array}$ & 1975 & 15 \\
\hline & & 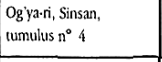 & 1 sépulure en jarres & 1985 & 6 \\
\hline & & $\begin{array}{l}\text { Wölsong.ni, Songsan, } \\
\text { tumulus n } n^{\circ} 1\end{array}$ & 1 unique sépulture en grandes jarres & $1985 ?$ & 10 \\
\hline & \multirow{3}{*}{$\begin{array}{l}\text { III' ou } \\
\text { début } \\
\text { IV siècle }\end{array}$} & $\begin{array}{l}\text { Wa' 'urti, 'Ka' kobun.gun, } \\
\text { uumuli no } 1 \text { et } 6\end{array}$ & $\begin{array}{l}1 \text { unique sépulure en grandes jarres } \\
\text { par tertre }\end{array}$ & 1986 & 7 \\
\hline & & $\begin{array}{l}\text { Wa'ven, 'Na' kobun-gun, } \\
\text { tumulus no } 1\end{array}$ & 1 unique sépulture en grandes jarres & 1986 & 7 \\
\hline & & $\begin{array}{l}\text { Ponghakkni, Sin'gorm, } \\
\text { tumulus supposć }\end{array}$ & 1 unique sépuluure en grandes jarres & 1987 & 20 \\
\hline & $\begin{array}{l}\text { débur } \\
\text { IV siecle }\end{array}$ & 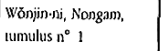 & $\begin{array}{l}2 \text { sípulures en grandes jares, } \\
\text { lertre supposé assez grand. }\end{array}$ & 1987 & 21 \\
\hline
\end{tabular}




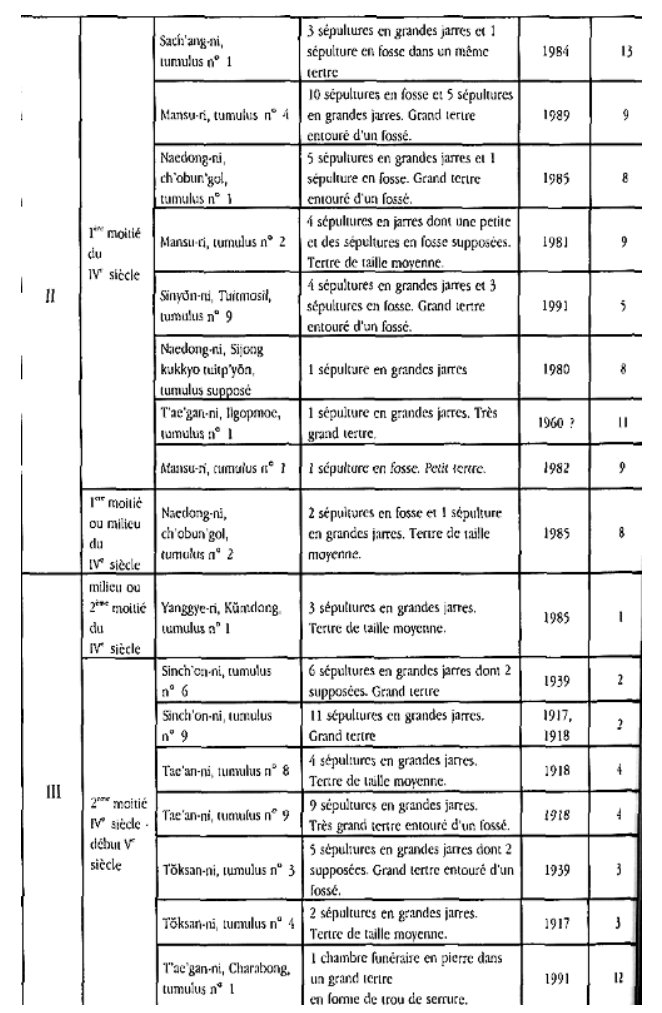

Tableau 2. Relations entre la forme de la base des céramiques et la technique de façonnage de la base (en nombre d'individus)

\begin{tabular}{|c|c|c|c|c|c|c|c|}
\hline \multirow{2}{*}{$\begin{array}{c}\text { Techniques de } \\
\text { façonnage de la base }\end{array}$} & \multicolumn{5}{|c|}{ Forme de la base des pots } & \multirow[b]{2}{*}{ Total } & \multirow[b]{2}{*}{ Pourcentage } \\
\hline & $\begin{array}{c}\text { plate } \\
\text { très large }\end{array}$ & $\begin{array}{l}\text { pointue- } \\
\text { arrondie }\end{array}$ & $\begin{array}{l}\begin{array}{l}\text { ronde- } \\
\text { aplatie }\end{array} \\
\end{array}$ & $\begin{array}{l}\text { plate ou } \\
\text { rentrante }\end{array}$ & $\begin{array}{c}\text { forme } \\
\text { inconnue }\end{array}$ & & \\
\hline Ajout d'un large fond plat & 14 & & & & & 14 & 6,6 \\
\hline $\begin{array}{l}\text { Façonnage de la base dans la } \\
\text { continuité de la panse }\end{array}$ & & 13 & 7 & 5 & 1 & 26 & 12,3 \\
\hline $\begin{array}{l}\text { Façonnage de la panse et de } \\
\text { la base en deux étapes } \\
\text { distinctes }\end{array}$ & & 8 & 31 & 34 & & 73 & 34,4 \\
\hline $\begin{array}{l}\text { Batage sur la base, panse } \\
\text { lissée }\end{array}$ & & & 12 & 3 & 1 & 16 & 7,5 \\
\hline Technique non identifiée & 26 & 1 & 34 & 7 & 15 & 83 & 39,2 \\
\hline Total & 40 & 22 & 84 & 49 & 17 & 212 & 100 \\
\hline
\end{tabular}


Tableau 3. Relations entre les motifs du battage et la technique de façonnage de la base (en nombre d'individus)

\begin{tabular}{|c|c|c|c|c|c|c|c|c|c|c|c|}
\hline \multirow{3}{*}{$\begin{array}{c}\text { Techniques de } \\
\text { façonnage de la base }\end{array}$} & \multicolumn{10}{|c|}{ Motifs imprimés par battage sur la panse ou éventuellement sur la base } & \multirow{3}{*}{ Total } \\
\hline & \multicolumn{3}{|c|}{ croisillons } & \multicolumn{4}{|c|}{ stries parallèles } & \multicolumn{2}{|c|}{ cordés } & \multirow{2}{*}{$?$} & \\
\hline & A & A+liss & $A+D$ & $\mathrm{Bl} / \mathrm{BII}+$ liss & $\mathrm{BI} / \mathrm{BII}+\mathrm{D}$ & BIII & BIII+liss & $\mathrm{C}+$ liss & $\mathrm{C}+\mathrm{D}+$ liss & & \\
\hline Ajout d'un large fond plat & 10 & & 2 & & 1 & & & 1 & & & 14 \\
\hline $\begin{array}{l}\text { Façonnage de la base dans } \\
\text { la continuité de la panse }\end{array}$ & 14 & 3 & 5 & & & & 1 & & 2 & 1 & 26 \\
\hline $\begin{array}{l}\text { Façonnage de la panse et de } \\
\text { la base en deux étapes } \\
\text { distinctes }\end{array}$ & 2 & 12 & 3 & 10 & 21 & 3 & 9 & 1 & 8 & 4 & 73 \\
\hline $\begin{array}{l}\text { Battage sur la base, } \\
\text { panse lissée }\end{array}$ & & 11 & & 1 & & & 1 & 3 & & & 16 \\
\hline Technique non identifiée & 2 & 13 & 3 & 1 & & & 1 & & & 63 & 83 \\
\hline Total & 28 & 39 & 13 & 12 & 22 & 3 & 12 & 5 & 10 & 68 & 212 \\
\hline
\end{tabular}

A : motifs de croisillons; $\mathrm{BI}$ : motifs de stries parallèles simples; $\mathrm{Bll}$ : motifs de stries parallèles recoupées par un ou plusieurs traits perpendiculaires; BIII : motifs de stries parallèles avec dessin en forme de patte d'oie; C : motif cordé; D : lignes horizontales lissées ou incisées sur les traces de battage. Liss : large zone lissée sur l'épaule; ? : décor incertain ou pas de traces de battage.

Tableau 4. Répartition des différentes techniques de façonnage de la base dans les trois groupes de sites étudiés (en nombre d'individus)

\begin{tabular}{|c|c|c|c|c|c|}
\hline \multirow{3}{*}{$\begin{array}{c}\text { Techniques de } \\
\text { façonnage de la base }\end{array}$} & \multicolumn{4}{|c|}{ Sites étudiés } & \multirow{3}{*}{ Total } \\
\hline & \multicolumn{2}{|c|}{ Groupe I : III's. } & \multirow{2}{*}{$\begin{array}{l}\text { Groupe II: } \\
1^{\text {trc }} \text { moitié IV's. }\end{array}$} & \multirow{2}{*}{$\begin{array}{c}\text { Groupe III : } \\
2^{\text {cme }} \text { moitié IV } \mathrm{V}^{*} \text { début } \mathrm{V}^{\mathrm{s}} \mathrm{s} .\end{array}$} & \\
\hline & Habitat & Sépultures & & & \\
\hline Ajout d'un large fond plat & 12 & 0 & 2 & 0 & 14 \\
\hline $\begin{array}{l}\text { Ajout d'un large fond plat supposé } \\
\text { (base/panse avec angulation) }\end{array}$ & 10 & 8 & 7 & 1 & 26 \\
\hline $\begin{array}{l}\text { Façonnage de la base dans la } \\
\text { continuité de la panse }\end{array}$ & 16 & 0 & 7 & 3 & 26 \\
\hline $\begin{array}{l}\text { Façonnage de la panse et de la base } \\
\text { en deux étapes distinctes }\end{array}$ & 19 & 5 & 16 & 33 & 73 \\
\hline $\begin{array}{l}\text { Battage sur la base, } \\
\text { panse lissée }\end{array}$ & 0 & 6 & 9 & 1 & 16 \\
\hline $\begin{array}{l}\text { Technique non identifiée } \\
\text { (base/panse sans angulation) }\end{array}$ & 5 & 11 & 20 & 21 & 57 \\
\hline Nombre total de céramiques & 62 & 14 & 62 & 58 & 212 \\
\hline
\end{tabular}

Tableau 5. Évolution des différentes techniques de façonnage de la base (en pourcentage du nombre de vases de chaque période pour lesquels la technique a pu être identifiée)

\begin{tabular}{|c|c|c|c|c|c|}
\hline \multirow[b]{2}{*}{ Sites étudiés } & \multicolumn{5}{|c|}{ Techniques de façonnage de la base } \\
\hline & $\begin{array}{l}\text { Ajout large } \\
\text { fond plat }\end{array}$ & $\begin{array}{l}\text { Ajout large fond plat } \\
\text { supposé }\end{array}$ & $\begin{array}{l}\text { Façonnage panse/ } \\
\text { base en continu }\end{array}$ & $\begin{array}{l}\text { Façonnage panse/ } \\
\text { base distinct }\end{array}$ & $\begin{array}{l}\text { Battage base, } \\
\text { panse lissée }\end{array}$ \\
\hline Groupe I : III's. & $16 \%$ & $24 \%$ & $21 \%$ & $32 \%$ & $8 \%$ \\
\hline Groupe II $: 1^{\text {tet }} 1 / 2 \mathrm{~F}^{\mathrm{s}} \mathrm{s}$ & $5 \%$ & $17 \%$ & $17 \%$ & $39 \%$ & $22 \%$ \\
\hline $\begin{array}{l}\text { Groupe III }: 2^{\text {eme }} 1 / 2 \mathrm{IV}^{*}- \\
\text { début } V^{*} \mathrm{~s} \text {. }\end{array}$ & $0 \%$ & $3 \%$ & $8 \%$ & $87 \%$ & $3 \%$ \\
\hline Remarques & \multicolumn{3}{|c|}{ Diminution progressive } & Augmentation & Peu significati \\
\hline
\end{tabular}


Tableau 6. Nombre de céramiques présentant des « accidents »

\begin{tabular}{|c|c|c|c|c|c|}
\hline \multirow[b]{2}{*}{ Problèmes rencontrés } & \multicolumn{3}{|c|}{ Sites étudiès } & \multirow[b]{2}{*}{ Total } & \multirow[b]{2}{*}{ Pourcentage } \\
\hline & $\begin{array}{l}\text { Groupe I : } \\
\text { III's. }\end{array}$ & $\begin{array}{l}\text { Groupe II : } \\
1^{\text {ste }} \text { moitié du IV's. }\end{array}$ & $\begin{array}{l}\text { Groupe III : } \\
2^{\text {cmec }} \text { moitiè du IV } \\
\text { début du } V^{*} s .\end{array}$ & & \\
\hline Déformations & 7 & 15 & 20 & 42 & 19,8 \\
\hline Cloques & 0 & 4 & 4 & 8 & 3,8 \\
\hline Couverte accidentellc & 0 & 4 & 2 & 6 & 2,8 \\
\hline Renfoncements & 1 & 10 & 12 & 23 & 10,8 \\
\hline Taches circulaires & 0 & 5 & 3 & 8 & 3,8 \\
\hline $\begin{array}{l}\text { Nombre de céramiques } \\
\text { défectueuses }\end{array}$ & 8 & 38 & 41 & 87 & \\
\hline $\begin{array}{l}\text { Nombre total de } \\
\text { céramiques }\end{array}$ & 92 & 62 & 58 & 212 & \\
\hline $\begin{array}{l}\text { Pourcentage de } \\
\text { céramiques défectueuses }\end{array}$ & 8,7 & 61,3 & 70,7 & 41 & \\
\hline
\end{tabular}

\section{BIBLIOGRAPHIE}

Barnes, Gina L.

1992. "The development of stoneware technology in southern Korea », pp. 197-208, in

C. M. Aikens et Rhee Song Nai [Yi Song-nae] (eds.), Pacific Northeast Asia in Prehistory: HunterFisher-Gatherers, farmers and Sociopolitical Elites. (Circum-Pacific Prehistory Conference, Seatle, Aug. 1989). Pullman : Washington State University Press.

Biagini, J. \& Mourer, R.

1971. « La poterie au Cambodge », Objets et Mondes 9 (2) : 200-220.

Ch'oe, Sŏng-nak

1993. Han'guk wŏnsamguk munhwa-ŭi yŏn'gu : Chŏnnam chibang-ŭi chungsim-ŭro. Séoul : Hagyŏn munhwasa.

Ch'oe, Pyŏng-hyŏn

1994. « Ch'ungbuk Chinch'ŏn Samnyong-ni, Sansu-ri-ŭi wŏnsamguk yoji-wa t'ogi », p. 117-131, in Han'guk hak hwan T'aep'yŏngyang kukche hoe-ŭi kogohak pumun [The Second Pacific Basin International Conference on Korean Studies], 2. Actes de la conférence internationale de Tokyo, juil. Tongbuk Asea kogohak yŏn'gu hoe.

Denès, Laurence

1995. «Les sépultures en grandes jarres du bassin du Yŏngsan-gang ", Arts Asiatiques 50 : 85-93 2000. « L'Âge du Fer dans le sud-ouest de la péninsule coréenne d'après les données archéologiques ", Arts Asiatiques 55 : 120-136.

Gallay, Alain, Huysecom, Eric \& Anne Mayor 1998. Peuples et céramiques du delta intérieur du Niger (Mali) : un bilan de cinq années de missions (1988-1993). Mainz am Rhein : von Zabern. 
Gibson, Alex \& Woods, Ann

1990. Prehistoric Pottery for the Archaeologist. Leicester/Londres/New York : Leicester University

Press.

Hamp'yŏng Wǒlgye-ri Sŏkkye kobun-gun II : 1991 nyŏndo palgul chosa.

1993. Séoul : Paekche munhwa kaebal yŏn'guwŏn/Kwangju : Chŏnnam taehakkyo pangmulgwan.

Ho, Chuimei

1984. « A bref survey of the pottery industry in villages in the south and in the north-east of Thailand », pp. 259-284, in J. Picton (ed.), Earthenware in Asia and Africa. Actes du Colloque des 21-23 juin 1982. Londres : Percival David Foundation.

Im, Yŏng-jin

1996. « Yŏngsan-gang yuyŏk-ŭi ihyŏng pun'gu », p. 21-34, in Honam chiyŏk kobun-ŭi pun'gu. Honam kogohak hoe haksul taehoe, 4. Actes du colloque de Kwangju, mars 1996. Kwangju : Honam kogohak hoe.

1993. Hamp’yŏng Wŏlgye-ri Sŏkkye kobun-gun I. Kwangju : Chŏnnam taehakkyo pangmulgwan.

Im, Yŏng-jin, Ch'oe, In-sŏn, Hwang, Ho-gyun \& Cho, Chin-sŏn

1995. Changsŏng Haksŏng-ni kobun-gun. Kwangju : Chŏnnam taehakkyo pangmulgwan.

Kim, Wŏn-yong

1986. Han'guk kogohak kaesŏl. Séoul : Ilchisa (3ème édition).

Kirk, Colin

1984. «Tradition or transformation ? : the Sinhalese potters of Ratmalaga-hawewa », pp. 1-27, in J. Picton (ed.), Earthenware in Asia and Africa. Actes du Colloque des 21-23 juin 1982. Londres :

Percival David Foundation.

Kroun, Eini

1984. " Methods of pottery manufacture among the Yami on Botel Tobago ", pp. 285-302, in

J. Picton (ed.), Earthenware in Asia and Africa. Actes du Colloque des 21-23 juin 1982. Londres :

Percival David Foundation.

Mourer, Roland

1984. « Technical progress, what for ? : some reflexions on pottery in Cambodia ", pp. 28-53, in

J. Picton (ed.), Earthenware in Asia and Africa. Actes du Colloque des 21-23 juin 1982. Londres :

Percival David Foundation.

Orton, Clive, Tyers, Paul \& Vince, Alan

1993. Pottery in Archaeology. Cambridge : University Press.

Pak, Chung-hwan

1996. Kwangju Myŏnghwa-dong kobun. Kwangju : Kungnip Kwangju pangmulgwan.

Pétrequin, Pierre \& Pétrequin, Anne-Marie

1999. «La poterie en Nouvelle-Guinée : savoir-faire et transmission des techniques », Journal de la Société des Océanistes 108 : 71-101.

Sanders, Herbert H. \& Kenkichi Tomimoto

1976. Connaître et réaliser la céramique japonaise. Fribourg : Office du Livre/Dessain et Tolra.

Simmonds, D.

1984. « Pottery in Nigeria », pp. 54-92, in J. Picton (ed.), Earthenware in Asia and Africa. Actes du Colloque des 21-23 juin 1982. Londres : Percival David Foundation.

Sŏ, Sŏng-hun \& Sŏng, Nak-chun

1986. Yŏngam Naedong-ni Ch'obun'gol kobun ; pu : Yŏngsan-gang yuyŏk-ŭi onggwanmyo chosa 
charyo. Kwangju : Kungnip Kwangju pangmulgwan.

1988. Naju Pannam kobun-gun : chonghap chosa pogosŏ. Kwangju : Kungnip Kwangju

pangmulgwan.

Sŏng, Nak-chun \& Kim, Kil-sik

1990. Yŏngam Mansu-ri 4 hobun. Kwangju : Kungnip Kwangju pangmulgwan.

« Terre africaine »

1994. Revue de la céramique et du verre 79 : 23-46.

Tite, M. S., Barnes, Gina L. \& C. Doherty

1992. «Stoneware Identification among Protohistoric Potteries of South Korea », pp. 64-69, in Science and Technology of Ancient Ceramics 2 (Proceedings of the International Symposium ISAC'92). Shanghai : Reaserch Society of Science and Technology of Ancient Ceramics.

Tobert, Natalie

1984. « Potters of El-Fasher : one technique practised by two ethnic groups », p. 219-237, in J. Picton (ed.), Earthenware in Asia and Africa. Actes du Colloque des 21-23 juin 1982. Londres : Percival David Foundation.

Van der Leeuw, Sander E.

1984. " Manufacture, trade and use of pottery on Negros, Phillipines ", pp. 326-364, in J. Picton (ed.), Earthenware in Asia and Africa. Actes du Colloque des 21-23 juin 1982. Londres : Percival David Foundation.

Woods, Ann 1984. " Methods of pottery manufacture in the Kavango region of Namibia : two case studies ", p. 303-325, in J. Picton (ed.), Earthenware in Asia and Africa. Actes du Colloque des 21-23 juin 1982. Londres : Percival David Foundation.

Yi, Sŏng-ju

1991. « Wŏnsamguk sidae t'ogi-ŭi yuhyŏng, kyebo, p'yŏnnyŏn, saengsan ch'eje », Han'guk kodaesa nonch'ong 2 : 235-298.

Yi, Yŏng-mun

1993. « Chŏnnam chibang sŏnsa sidae yŏn'gu sa : yŏn'gu sŏnggwa-wa kwaje », Chŏnnam sahak 7 : 9-48.

\section{NOTES}

1. L'aboutissement de ce travail a été rendu possible grâce au soutien financier de la Fondation Singer-Polignac que je tiens à remercier ici très sincèrement. Mes remerciements s'adressent également à Mme Sophie Méry et MM. Claude Constantin, Pierre Pétrequin et Alain Thote, qui ont accepté de relire ce texte et m'ont fait part de leurs commentaires.

2. Pour plus de détails sur l'Âge du Fer dans le sud-ouest de la péninsule coréenne, voir

L. Denès (2000).

3. Deux autres royaumes (dits de Silla et de Kaya) s'établissent aussi à peu près à la même période dans le sud-est de la péninsule.

4. Les rapports de fouilles consultés pour les Ve-VIe siècles sont les suivants : Kwangju Wŏlchŏn-dong (atelier de potier (?), début du Ve siècle : cf. Im Yŏng-jin, 1996); Kwangju Myŏnghwa-dong (tumulus en forme de trou de serrure à chambre funéraire en pierre, début du VIe siècle : $c f$. Pak Chung-hwan, 1996); Hamp'yŏng Wŏlgye-ri Sŏkkye (groupe 
de chambres en pierre, fin du Ve, début du VIe siècle : cf. Im Yŏng-jin, 1993 et Hamp'yŏng Wŏlgye-ri..., 1993); Changsŏng Haksŏng-ni (groupe de chambres en pierre, Ve-VIe siècles : $c f$. Im Yŏng-jin et al., 1995). J'ai également pu observer une partie des céramiques du tumulus à chambre en pierre de Haenam Wŏlsong-ni (Ve-VIe siècles) au musée national de Kwangju.

5. L'exemple donné concerne exclusivement de grands pots pour brasser la bière (contenance de 20 à 35 litres environ), quasi sphériques; mais il semble que cette remarque soit également pertinente à titre plus général.

6. Cette idée m'avait été suggérée par $\mathrm{S}$. van der Leeuw, que je remercie à cette occasion, et il semble effectivement qu'elle ne doive pas être négligée. On peut également citer l'exemple du Nigeria où les pots avec des traces de coups de feu (survenus lors de leur cuisson) sont appréciés et vendus un peu plus chers que les autres (Simmonds 1984, légende de la fig. 14b, p. 72 et photo p. 87).

\section{RÉSUMÉS}

La technique du battage est attestée dans le sud-ouest de la Corée à partir du début de notre ère, mais les modalités de sa diffusion et de son développement restent mal connues. Afin de poser les bases d'une meilleure compréhension de cette technique, sont analysées, à partir d'un échantillon de céramiques des IIIe-IVe siècles, les traces imprimées en surface par le battoir gravé ou entouré de cordes. De grandes lignes peuvent être dégagées: origine, évolution, changements occasionnés, relations avec les nouveaux types de four. Cette première approche, dépendant des données archéologiques disponibles, permet de distinguer plusieurs types de battage, et reflète ainsi un phénomène complexe, difficile à interpréter de façon linéaire.

The use of beating in the making of corean ceramics during the Iron age. The technique of beating has existed in southwest Corea since the beginning of our era, but the way it has spread and develop are not well known. For a better understanding of this technique we analyse, through a sample of III-Ivth centuries ceramics the marks left by the beater, engraved or marked by ropes. We can thus bring out the origine, evolution, changes of this technique and how it is linked with new types of oven. This first approach, based on the available archaeological data, allows to distinguish several ways of beating, revealing a complex phenomenon, which is hard to interprate in terms of linear evolution.

El batimiento en la fabricación de las cerámicas coreanas de la Edad del Hierro. La técnica del batimiento es atestiguada en el Suroeste de Corea desde los principios de nuestra era, pero las modalidades de su difusión y de su desarrollo quedan poco conocidas. Para establecer las bases de una mejor comprensión de esta técnica, la autora analiza, en una muestra de cerámicas de los siglos III y IV, las huellas dejadas por la paleta grabada o encordada. Así se pueden esbozar el origen, la evolución, los cambios, las relaciones con nuevos tipos de hornos. Esta primera aproximación permite diferenciar varios tipos de batimiento y refleja un fenómeno complejo, dificil de interpretar linealmente. 
INDEX

Mots-clés : poterie, battage, céramique, Corée du Sud, fours

AUTEUR

LAURENCE DENÈS

Laboratoire d'Études coréennes, UMR 8033-CNRS 